Radiologe $2021 \cdot 61: 1028-1030$

https://doi.org/10.1007/s00117-021-00914-3

Angenommen: 24. August 2021

Online publiziert: 6. Oktober 2021

(c) Springer Medizin Verlag $\mathrm{GmbH}$, ein Teil von Springer Nature 2021

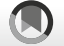

Check for
updates

\section{Radiologie auf modernen (Ab)wegen}

Lisa Ullrich

Diagnostische und Interventionelle Radiologie und Neuroradiologie, München Klinik Bogenhausen, München, Deutschland
In diesem Artikel wird aus Gründen der besseren Lesbarkeit das generische Maskulinum verwendet. Weibliche und anderweitige Geschlechteridentitäten werden dabei ausdrücklich mitgemeint, soweit es für die Aussage erforderlich ist.

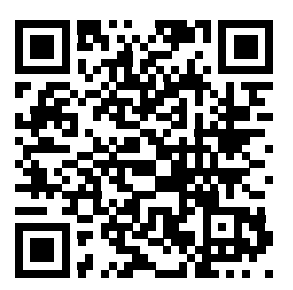

QR-Code scannen \& Beitrag online lesen

\section{Pandemiebedingte Veränderung der Arbeitswelt}

„Homeoffice, wann immer es geht ${ }^{\prime}$ ist von der Politik zuletzt Anfang Januar dieses Jahres zur weiteren Eindämmung der COVID-19-Pandemie gefordert worden. Mittels vermehrter Heimarbeit sollten durch die Ausdünnung von Teams etwa in Büros Ansteckungsrisiken minimiert werden. Die globale Pandemie zeigt, wie wichtig Flexibilität bzw. eine schnelle Anpassung der Arbeitsverhältnisse ist, etwa um Menschen vor einer Infektion im beruflichen Umfeld bestmöglich zu schützen.

Durch die Pandemie und die in ihrer Folge ausgelöste Debatte um flexible Heimarbeit wurde allerdings nur noch einmal auf ein Thema aufmerksam gemacht, das auch in anderem Kontext bereits seit geraumer Zeit präsent ist: Zahlreiche Ärztinnen, aber auch immer mehr junge Ärzte wollen zwecks Kinderbetreuung zumindest für eine gewisse Zeit in Teilzeitmodellen arbeiten.

Die Medizin wird zum einen immer weiblicher [1-3] - ein Trend, der in den letzten Jahren stetig zugenommen hat. Zum anderen teilen sich immer mehr Paare die Kinderbetreuung fair auf; beide Partner streben einen raschen Wiedereinstieg in das Berufsleben an oder wollen im besten Fall gar nicht aussteigen.

\section{Homeoffice für Radiologen}

Während die Technik, sei es etwa in Bezug auf Qualität oder Innovation der Untersuchungsmodalitäten/-geräte, Befundungsprogramme und künstliche Intelligenz in den letzten Jahren beachtliche Fortschritte vorweisen kann, hat sich das Berufsbild des praktizierenden Radiologen nur langsam entwickelt.

Obwohl die Radiologie in vielen Teilgebieten keinen direkten Patientenkontakt erfordert und zudem ein relevanter Anteil der radiologischen Diagnostik keiner sofortigen Befunderstellung bedarf, finden sich bisher nur wenige Beispiele dafür, dass Radiologinnen und Radiologen zum Beispiel flexibel von zu Hause aus Befunde erstellen.

Zwar wurde an einigen Kliniken und Praxen in den letzten Monaten im Rahmen der Pandemie verstärkt der Nachfrage nach Homeoffice nachgegangen, eine wirklich flächendeckend vermehrte Einrichtung von Heimarbeitsplätzen hat jedoch nicht stattgefunden.

Mit einer großzügigen Einführung von Heimcomputern könnte man den in Teilzeit arbeitenden Kollegen ein weitaus flexibleres Arbeitsmodell anbieten, ohne an einen starren Zeitrahmen gebundene Präsenzpflicht. Damit könnten Ausfälle von Arbeitskräften, etwa aufgrund von Krankheit der Kinder oder Kitaschließung potenziell vermieden werden. Auch wäre die Möglichkeit, junge Eltern früh wieder in die klinische Arbeit einzubinden, besser gegeben.

Der formelle Rahmen für das „Homeoffice für Radiologen" ist durch das Strahlenschutzgesetz klar vorgegeben: Es muss gemäß § 83 bzw. § 14 StrlSchG im Klinikalltag gewährleistet sein, dass am Ort der technischen Durchführung der Untersuchung mindestens ein Arzt mit der erforderlichen Fachkunde die rechtfertigende Indikation stellt oder aber ein Arzt mit den erforderlichen Kenntnissen im Strahlenschutz die zur Feststellung der rechtfertigenden Indikation erforderlichen Angaben ermittelt und prüft sowie in Abstimmung mit dem 
fachkundigen Arzt im Homeoffice die Patientenaufklärung übernimmt (Teleradiologie). Hinsichtlich der Aufteilung wäre dann denkbar, dass Notfalluntersuchungen beispielsweise von den Kollegen vor Ort betreut und auch befundet werden. Elektive Untersuchungen könnten - ohne Notwendigkeit einer unmittelbaren Befundung von den jeweiligen Homeoffice-Kollegen abgearbeitet werden.

\section{Wie also könnte das Ganze umgesetzt werden?}

Bauliche und rechtliche Anforderungen müssten hier selbstverständlich erfüllt werden. Zunächst wäre initial zu prüfen, ob der vorgesehene Arbeitsplatz daheim überhaupt für eine Homeoffice-Einrichtung geeignet ist.

Folgende Gesichtspunkte zur Schaffung eines adäquaten Arbeitsplatzes wären hier beispielsweise zu beachten:

- Einstellungsmöglichkeiten von Bürostuhl und Arbeitstisch zueinander (wie auch im Klinikalltag),

- eine korrekte Position der Befundungsmonitore,

- der Abstand zu den Monitoren,

- die Vermeidung von Lärmquellen,

- eine angepasste Beleuchtung.

Gerade die anpassbare Raumhelligkeit ist bekanntermaßen für die radiologische Befundung essenziell, da die Umgebungsleuchtdichte die Leuchtdichte etwa eines Röntgenbildes aufgrund einer anteiligen Reflexion durch die Befundungsoberfläche beeinflusst. So müssen blendende Bereiche um die Bildschirmfläche abgedunkelt werden können (Fenster o. Ä.) respektive ein Fremdlichteinfluss ausgeschlossen sein. Zudem wäre unbedingt zu beachten, dass sich kein Dritter Zugang zu dem Arbeitsrechner verschaffen kann. Dies läge letztlich in der Verantwortung des jeweiligen Arztes. Analog zu einem VPN-Client könnte die Datenübertragung über eine sichere Leitung mittels des privaten Internetanschlusses des jeweiligen Arztes laufen. Die Kommunikation zwischen Homeoffice und Klinik wäre somit geschützt. Es wäre sichergestellt, dass die Patientenbilder in dem Rechenzentrum des Arbeitgebers verbleiben. Die sensiblen Daten könnten also nicht heruntergeladen werden. Moni- tore und Rechner würden vom Arbeitgeber gestellt und zu Beginn vom Hersteller abgenommen werden; zusätzlich müssten nach DIN natürlich regelmäßig - wie man das aus dem Klinikalltag kennt - Konstanzprüfungen durchgeführt werden. ${ }^{1}$

Alles in allem wäre eine HomeofficeEinrichtung zunächst mit Mehraufwand verbunden und würde für den Arbeitgeber eine zusätzliche Investition bedeuten. Sie ist aber aus den o.g. Gesichtspunkten als eine sinnvolle und letztlich logische Entwicklung für den modernen Arbeitsalltag als Radiologe anzusehen.

\section{Alternative Arbeitsmodelle}

Neben örtlich flexiblen Modellen einschließlich der Förderung von Arbeit im Homeoffice entwickeln sich zumindest vereinzelt auch Modelle für eine radiologisch ärztliche Tätigkeit abseits der klassischen Dichotomie Klinik/Praxis, Anstellung/Partnerschaft.

Innovative Beispiele hierfür finden sich etwa bei Praxisverbünden oder Netzwerken mit Genossenschaftscharakter aus niedergelassenen und freischaffenden Radiologen mit dem Versprechen einer weiteren Flexibilisierung der Arbeitszeit. Das soll etwa dadurch erreicht werden, dass die Radiologen in Eigenregie an ihre jeweiligen Arbeitszeiten angepasste Untersuchungstermine vergeben. Vor allem bei Radiologen mit Familie sei dies ein sehr beliebtes Konzept, wie in einem Telefoninterview berichtet wird. Teils werden sogar ausschließlich teleradiologische Arbeitsmöglichkeiten für die angestellten Radiologen angeboten. Inwieweit solche Modelle aus der ambulant und elektiv arbeitenden Praxis in den Alltag einer Klinik übertragbar sind, in dem die interdisziplinäre Diskussion und der Austausch mit den klinischen Partnerdisziplinen von essenzieller Bedeutung ist, bleibt allerdings bis dato offen.

Um Radiologie für qualifizierten Nachwuchs langfristig attraktiv zu halten, werden zukünftig weitere, fortschrittliche Konzepte für die radiologische Tätigkeit entwickelt und innovative, moderne Arbeitsmodelle etabliert werden müssen; wenige andere Fächer bieten in vergleichbarem

1 Telefoninterview mit Mitarbeitern der IT und des Datenschutzes der München KlinikgGmbH.
Maß die Chance, flexibel an die Lebensumstände der ärztlichen Mitarbeitenden angepasst zu werden.

\section{Fazit}

- Es sollten Forderungen nach einem flächendeckenden, zumindest anteiligen Homeoffice nicht nur im Rahmen der Pandemie, sondern auch für Teilzeitkräfte und/oder junge Mütter gestellt werden. Stichwort: sowohl Schutz der Gesundheit, Vereinbarkeit von Beruf und Familie als auch effektive Nutzung der Ressourcen.

- Moderne Alternativen zur radiologischen Tätigkeit in Klinik und Praxis sind zwar noch selten, es gibt aber schon vereinzelt innovative Vorreiter.

\section{Korrespondenzadresse}

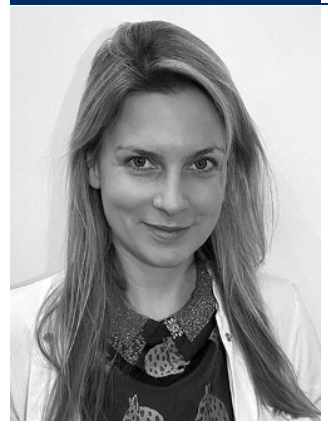

\section{Dr. med. Lisa Ullrich}

Diagnostische und Interventionelle Radiologie und Neuroradiologie, München Klinik Bogenhausen

Englschalkinger Str. 77, 81925 München,

Deutschland

lisa.ullrich@muenchen-klinik.de

\section{Einhaltung ethischer Richtlinien}

Interessenkonflikt. L. Ullrich gibt an, dass kein Interessenkonflikt besteht.

Für diesen Beitrag wurden vom Autor keine Studien an Menschen oder Tieren durchgeführt. Für die aufgeführten Studien gelten die jeweils dort angegebenen ethischen Richtlinien.

\section{Literatur}

1. Statista (2021) Anzahl der Studierenden im Fach Humanmedizin in Deutschland nach Geschlecht in den Wintersemestern von 2007/2008 bis 2019/2020. https://de.statista.com/statistik/ daten/studie/200758/umfrage/entwicklung-deranzahl-der-medizinstudenten/. Zugegriffen: Juni 2021

2. Statista (2021) Anzahl der berufstätigen Ärztinnen in Deutschland nach Arztgruppe in den Jahren 2016 bis 2020. https://de.statista.com/ 
statistik/daten/studie/158852/umfrage/anzahlder-aerztinnen-nach-taetigkeitsbereichen/. Zugegriffen: Juni 2021

3. Bundesärztekammer (2021) Berufstätige Ärzte. https://www.bundesaerztekammer.de/ ueber-uns/aerztestatistik/aerztestatistik-2019/ berufstaetige-aerzte/.Zugegriffen:Juni 2021

\section{Dunkelfeld-Röntgen verbessert Diagnose von Lungenerkran- kungen}

\section{Neue Röntgentechnologie im Patienteneinsatz}

Forschende der Technischen Universität München (TUM) haben ein neues Röntgenverfahren für die Lungendiagnostik erstmalig erfolgreich bei Patienten eingesetzt. Dunkelfeld-Röntgen macht frühe Veränderungen in der Alveolarstruktur sichtbar, benötigt dafür jedoch nur ein Fünfzigstel der in der Computertomographie üblichen Strahlendosis.

Millionenfach führen schwere Erkrankungen des Atmungssystems zu stark eingeschränkter Lebensqualität. Jedes Jahr sterben allein in Deutschland mehr als 100.000 Menschen an schweren Lungenerkrankungen. Typisch für eine lebensgefährliche COPD sind teilweise zerstörte Lungenbläschen und ein Emphysem.

In normalen Röntgenaufnahmen sind die feinen Unterschiede im Gewebe jedoch kaum sichtbar. Detaillierte diagnostische Informationen liefern erst fortschrittliche medizinische Bildgebungstechnologien, bei denen im Computer viele Einzelbilder zusammengesetzt werden. Eine schnelle und kostengünstige Option mit geringer Strahlenbelastung für Früherkennung und Nachuntersuchungen fehlt bisher.

Diese Lücke könnte ein an der TU München entwickeltes Verfahren schließen: das DunkelfeldRöntgen. In der aktuellen Ausgabe von „Lancet Digital Health“ präsentiert ein Forschungsteam, angeführt von Franz Pfeiffer, Professor für biomedizinische Physik und Direktor des Munich Institute of Biomedical Engineering der TUM, nun Ergebnisse einer ersten klinischen Studie mit Patienten, bei der die neue Röntgen-Technologie zur Diagnose der Lungenkrankheit COPD eingesetzt wurde.

\section{Der Wellencharakter des Röntgenlichts macht's möglich}

Die konventionelle Röntgen-Bildgebung beruht auf der Abschwächung des Röntgenlichts auf seinem Weg durch das Gewebe. Die Dunkelfeld-Technologie dagegen nutzt Anteile des Röntgenlichts, die gestreut werden und beim konventionellen Röntgen unbeachtet bleiben. Die neue Methode nutzt damit das physikalische Phänomen der Streuung auf ähnliche Weise wie die schon länger bekannte Dunkelfeldmikroskopie mit sichtbarem Licht: Diese macht es möglich, Strukturen weitgehend transparenter Objekte sichtbar zu machen. Im Mikroskop erscheinen sie als helle Strukturen vor einem dunklen Hintergrund, was der Methode ihren Namen verleiht.

\section{Geringere Strahlendosis}

Eine Untersuchung mit der Dunkelfeld-Röntgen-Technik ist außerdem mit einer deutlich geringeren Strahlendosis verbunden als die heute verwendete Computertomografie. Denn sie erfordert nur eine einzelne Aufnahme pro Patientin oder Patient, während für die Computertomografie zahlreiche Einzelaufnahmen aus verschiedenen Richtungen erstellt werden müssen. Darüber hinaus haben die ersten klinischen Ergebnisse bestätigt, dass das Dunkelfeld-Röntgen zusätzliche bildliche Informationen über die zugrundeliegende Mikrostruktur der Lunge liefert. Das Dunkelfeld-Röntgen könnte so zu einer besseren Früherkennung von COPD und anderen Lungenerkrankungen in Zukunft beitragen

\section{Literatur:}

Willer K, Fingerle A, Noichl W et al (2021) X-ray dark-field chest imaging for detection and quantification of emphysema in patients with chronic obstructive pulmonary disease: a diagnostic accuracy study. Lancet Digital Health, 3 (11) e733-e744, DOI: 10.1016/S25897500(21)00146-1 